\title{
Mandenkan
}

MANDENIKAN Bulletin semestriel d'études linguistiques mandé

$50 \mid 2013$

Numéro 50

\section{Le comportement tonal des marqueurs prédicatifs dans la langue kakabé}

The tonal behavior of predicative markers in the Kakabe language

ТОНАЛЬНОЕ ПОВЕДЕНИЕ ПРЕДИКАТИВНЫХ ПОКАЗАТЕЛЕЙ В ЯЗЫКЕ КАКАБЕ

\section{Alexandra Vydrina}

\section{OpenEdition}

Journals

Édition électronique

URL : https://journals.openedition.org/mandenkan/283

DOI : $10.4000 /$ mandenkan.283

ISSN : 2104-371X

\section{Éditeur}

Llacan UMR 8135 CNRS/Inalco

Édition imprimée

Date de publication : 1 décembre 2013

Pagination : 147-170

ISSN : 0752-5443

\section{Référence électronique}

Alexandra Vydrina, « Le comportement tonal des marqueurs prédicatifs dans la langue kakabé », Mandenkan [En ligne], 50 | 2013, mis en ligne le 01 décembre 2013, consulté le 08 juillet 2021. URL http://journals.openedition.org/mandenkan/283 ; DOI : https://doi.org/10.4000/mandenkan.283

Ce document a été généré automatiquement le 8 juillet 2021.

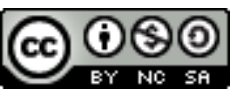

Les contenus de Mandenkan sont mis à disposition selon les termes de la Licence Creative Commons Attribution - Pas d'Utilisation Commerciale - Partage dans les Mêmes Conditions 4.0 International. 


\section{Le comportement tonal des marqueurs prédicatifs dans la langue kakabé}

The tonal behavior of predicative markers in the Kakabe language

ТОНАЛЬНОЕ ПОВЕДЕНИЕ ПРЕДИКАТИВНЫХ ПОКАЗАТЕЛЕЙ В ЯЗЫКЕ КАКАБЕ

\section{Alexandra Vydrina}

\section{NOTE DE L'AUTEUR}

Cette recherché a été menée avec le soutien financier de la fondation ELDP, subvention IGSO197 « Description and the documentation of the Kakabe language » et de la Fondation russe des sciences humaines, subvention 13-34-01015 « Les systèmes verbaux en langues mandé dans le contexte typologique et la recherche régionale ».

\section{Abréviations}

$\mathrm{B}=$ ton bas

$\mathrm{ART}=$ article référentiel

CAUS = causatif

$\mathrm{FH}=$ futur-habituel

GER = gérondif

$\mathrm{H}=$ ton haut

LOC = locatif

$\mathrm{MP}$ = marqueur prédicatif

NEG = négation

$\mathrm{PFV}=$ perfectif

$\mathrm{PL}=$ pluriel

POSS = marqueur possessive

PC.RES = participe résultatif

PST.INTR = prétérit dans la construction intransitive 
PST.TR = prétérit dans la construction transitive

$\mathrm{PRF}=$ parfait

$\mathrm{REF}=$ réfactif

REFL $=$ réflexif

$\mathrm{SEQ}=$ séquentiel

$S G=$ singulier

VERB.PL = pluralité verbale.

\section{Introduction}

1 Cet article concerne la question de la réalisation des tons sur les auxiliaires dans la langue kakabé (mokolé < mandé ouest) et, dans une perspective plus large, celle du rapport entre les tons lexicaux et grammaticaux ainsi que celle de l'interaction entre les tons des morphèmes dans l'énoncé ${ }^{\text {. }}$

2 Le Kakabé est une langue minoritaire de la République de Guinée parlée sur le plateau du Fouta-Jalon. Il est difficile d'évaluer le nombre de locuteurs du kakabé parce qu'ils n'ont jamais été pris en compte dans les recensements : face à des étrangers, les Kakabé s'identifient souvent avec les Peuls. Quant à la variation dialectale, les différences les plus importantes sont attestées entre les variétés parlées dans la sous-préfecture de Timbo (dialectes sud-est) et les variétés parlées dans la sous-préfecture de Kankalabé (dialectes nord-ouest).

3 Comme pratiquement toutes les langues mandé, le kakabé est une langue tonale. Est attestée une opposition de deux tons, haut et bas. Normalement, dans une langue tonale, à chaque syllabe est attribuée un des tons. En même temps, les mécanismes d'attribution des tons de surface pour chaque syllabe sont différents en fonction de divers paramètres.

4 L'ordre des mots en kakabé est strictement $\mathrm{S}-\mathrm{Aux}-\mathrm{O}-\mathrm{V}-\mathrm{X}$ avec le $\mathrm{X}$ référant à tout constituant sauf sujet ou complément d'objet direct.

5 Les valeurs d'aspect, de temps, de polarité et de mode sont exprimées cumulativement par le paradigme d'auxiliaires qui sont appelés dans la tradition mandéisante les marqueurs prédicatifs (MP). Leur position syntaxique dans l'énonce est strictement fixe : immédiatement après le groupe sujet, par exemple :

\begin{tabular}{|c|c|c|c|c|}
\hline (1a) & $\grave{n}$ & bátí & no-"inge & sàn \\
\hline & $1 \mathrm{SG}$ & PRF & lait.ART & acheter \\
\hline & & & du lait'. & \\
\hline
\end{tabular}

\begin{tabular}{|l|l|l|l|l|}
\hline (1b) & $\grave{n}$ & máá & nJ:-ne & sàn \\
\hline & 1SG & NEG.PRF & lait.ART & acheter \\
\hline \multicolumn{4}{|l}{} \\
\multicolumn{4}{|l}{ 'Je n'ai pas acheté de lait'. } \\
\hline
\end{tabular}


Le Tableau 1 ci-dessous présente le paradigme des marqueurs TAM et de la polarité en kakabé. Toutes ces valeurs prédicatives sont exprimées par les MP (dans le cas du progressif affirmatif et négatif, un MP se combine avec le suffixe verbal du gérondif). La seule exception est le prétérit intransitif, exprimé par le suffixe verbal -ta.

Tableau 1. Le paradigme des marqueurs TAM et de polarité en kakabé

\begin{tabular}{|l|l|}
\hline MP affirmatifs & MP négatifs \\
\hline bi ...-la - progressif (MP avec un suffixe de & bélé ...-la - progressif négatif (MP avec un suffixe de \\
gérondif) & gérondif) \\
bátí- parfait & máá - perfectif négatif \\
$k a-$ prétérit transitif & tée - futur-habituel négatif \\
-ta-prétérit intransitif (suffixe verbal) & kání-impératif négatif \\
$n i-$ modal & \\
si-futur-habituel & \\
mání- conditionnel & \\
\hline
\end{tabular}

1. La série des MP négatifs est réduite par rapport au nombre des MP affirmatifs. Premièrement, dans la série négative il n'y a pas d'opposition entre le parfait et le prétérit. En conséquent, c'est le perfectif négatif qui s'oppose au parfait comme au prétérit affirmatif. Deuxièmement, il n'y a pas de marqueur négatif du conditionnel.

2. Le marqueur du progressif (soit négatif, soit affirmatif) se distingue des autres membres du paradigme par le fait qu'il est exprimé par la combinaison de l'auxiliaire bi (pour l'affirmatif) ou bele (pour le négatif) et du suffixe verbal -la, (tandis que les autres MP ne contiennent qu'un seul morphème) :

\begin{tabular}{|l|l|l|l|l|}
\hline (2a) & Fa'-íntá & bíi & bàntàráa & tùgú-lá \\
\hline & Fanta & être & manioc.ART & piler-GER \\
\hline & \multicolumn{4}{|l}{ 'Fanta est en train de piler le manioc'. } \\
\hline
\end{tabular}

\begin{tabular}{|l|l|l|l|l|l|}
\hline$(2 b)$ & Fa'́ntá & bélé & bàntàráa & tùgú-lá & \\
\hline & Fanta & être.NEG & manioc.ART & piler-GER & \\
\hline & \multicolumn{4}{|c|}{ 'Fanta n'est pas en train de piler le manioc'. } \\
\hline
\end{tabular}

3. L'expression du prétérit dépend de la transitivité/intransitivité de la construction. Il est marqué par l'auxiliaire $k a$ dans les propositions intransitives et par le suffixe -ta dans les propositions transitives:

\begin{tabular}{|l|l|l|l|l|l|l|}
\hline (3) & àn & tágá-tá, & àn & ká & nc̀téc̀-nù & yén \\
\hline & 3PL & aller-PST.INTR & 3PL & PST.TR & noix.ART-PL & voir \\
\hline
\end{tabular}




\begin{tabular}{|c|c|c|c|c|c|}
\hline àn & $y \varepsilon \grave{c} l \varepsilon ́-t a ́$ & àn & $k a ́$ & 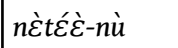 & bóó \\
\hline $3 \mathrm{PL}$ & monter-PST.INTR & 3PL & PST.TR & noix.ART-PL & sortir \\
\hline
\end{tabular}

7 Quant au comportement tonal d'un MP, il dépend, d'abord, de sa forme phonologique et, ensuite, du contexte. Ces deux paramètres sont analysés en détail dans les sections 1,2 et 3 . La section 4 concerne la question plus générale du rapport entre la grammaticalité du morphème, sa longueur et sa capacité à porter son propre ton.

\section{Les MP dans la position devant le verbe ou devant le nom}

\subsection{La polarisation du ton}

8 Le comportement tonal des MPs dépend de deux paramètres phonotactiques. Comparons le ton d'un MP monosyllabique et d'un MP dissyllabique lorsqu'ils sont devant un nom à ton haut (pour rendre la présentation plus explicite, une ligne supplémentaire est mise sous la ligne des gloses où les tons sont désignés par les lettres, $\mathrm{H}$ pour le haut, B pour le bas) :

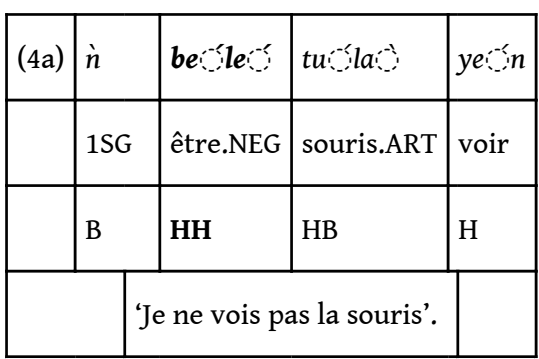

\begin{tabular}{|l|l|l|l|l|}
\hline (4b) & $\grave{n}$ & si & tuila & yeín \\
\hline & 1SG & FH & souris.ART & voir \\
\hline & B & B & HB & H \\
\hline \multicolumn{4}{|l}{} \\
\hline
\end{tabular}

9 D'après ces deux exemples on pourrait conclure que le MP bélé est de ton haut et le MP si de ton bas. Cependant, considérons le cas où la position du complément d'objet direct est occupée par un nom à ton bas initial :

\begin{tabular}{|c|c|c|c|c|}
\hline (5a) & $\grave{n}$ & be & sùsée & yer-in \\
\hline & $1 S G$ & NEG.IPFV & poulet.ART & voir \\
\hline
\end{tabular}




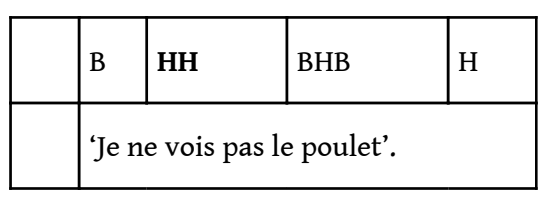

\begin{tabular}{|l|l|l|l|l|}
\hline$(5 \mathrm{~b})$ & $\grave{n}$ & sí & sùsée & yeín. \\
\hline & $1 \mathrm{SG}$ & $\mathrm{FH}$ & poulet.ART & voir \\
\hline & B & H & BHB & $\mathrm{H}$ \\
\hline \multicolumn{4}{|l}{} \\
\multicolumn{4}{|l}{ 'Je verrai le poulet'. } \\
\hline
\end{tabular}

10 Le ton du MP bélé est le même que dans la phrase (2). Par contre, le ton de si est haut, contrairement à ce qu'on observe dans la phrase (3). Dans les deux cas, le ton de si est différent du ton de la syllabe suivante : haut devant le bas et bas devant le haut. Cela permet de conclure que le ton inhérent de bélé est haut et que si n'a pas de son propre ton, ou plutôt que ce MP est caractérisé par un ton qui se polarise par rapport au ton de la syllabe suivante.

11 Afin de déterminer les tons de toutes les marques prédicatives kakabé, chacune a été enregistrée dans deux contextes (comme dans les exemples 2-5), devant un nom à ton haut et devant un nom à ton bas :

\begin{tabular}{|l|l|l|l|l|}
\hline$(6 \mathrm{a})$ & $\grave{n}$ & MP & tui la & yeín \\
\hline$(6 \mathrm{~b})$ & $\grave{n}$ & MP & sùsè & ye \\
\hline
\end{tabular}

12 Ont été également examinées presque toutes les occurrences de chaque MP dans le corpus de textes.

Le résultat de l'analyse est que les MP d'une seule syllabe légère se caractérisent par un ton polarisant. Voici ces MP avec leurs valeurs données entre parenthèses :

$k a-$ (prétérit intransitif) ;

$n i$ - (optatif);

$s i$ - (futur-habituel) ;

$b i$ - (MP qui en combinaison avec le suffixe verbal -la indique le progressif).

13 Le marqueur du séquentiel ka ne comporte qu'une seule more, mais il est de ton bas dans n'importe quel contexte :

\begin{tabular}{|l|l|l|l|l|l|l|}
\hline (7) & à & táá-tá & kà & jíídìisá & filá & nààti. \\
\hline & 3SG & aller-PST.INTR & SEQ & oiseau & deux & apporter \\
\hline \multicolumn{6}{|l}{} \\
\multicolumn{4}{|l}{ 'Il est parti et a rapporté deux oiseaux'. } \\
\hline
\end{tabular}

\begin{tabular}{|l|l|l|l|l|l|l|}
\hline (8) & síikúlí-ndè & bòyíttá, & kà & wúlí, & kà & pàtápàtà. \\
\hline
\end{tabular}




\begin{tabular}{|l|l|l|l|l|l|l|}
\hline & bouc-DIM.ART & tomber-PST.INTR & SEQ & se.lever & SEQ & se.débattre \\
\hline & 'Le petit bouc est tombé, puis il s'est levé, puis il a commencé à se débattre'. \\
\hline
\end{tabular}

Bien que le marqueur ka soit toujours placé devant le groupe verbal, il est syntaxiquement différent des MP. Il n'apparaît en effet que dans les énoncés où le sujet n'est pas exprimé, comme dans les exemples (7) et (8). En outre, la valeur sémantique du séquentiel exprimée par kaì ne fait pas partie des catégories de TAM et de polarité exprimées par les MP. On reviendra sur ce marqueur et son ton bas stable dans la section 4 qui porte sur le rapport entre la grammaticalité du morphème, sa dimension et sa capacité à porter son propre ton.

Les exemples suivants montrent la polarisation du ton des MP monomores $k a, n i$, si et $b i$ par rapport au ton du nom suivant :

\begin{tabular}{|l|l|l|l|l|l|}
\hline$(9)$ & $\grave{j}$ & nì & síkkè & lè & sàà. \\
\hline & $2 \mathrm{PL}$ & OPT & chanson.ART & FOC & mettre \\
\hline & \multicolumn{5}{|c|}{ 'Vous devez chanter une chanson'. } \\
\hline
\end{tabular}

\begin{tabular}{|c|c|c|c|c|}
\hline (10) & j̀ & $n i i^{-\zeta}$ & jàgáà & da:a. \\
\hline & $2 \mathrm{PL}$ & OPT & nid.ART & fabriquer \\
\hline & \multicolumn{4}{|c|}{ 'Vous devez fabriquer un nid'. } \\
\hline
\end{tabular}

\begin{tabular}{|l|l|l|l|l|l|l|}
\hline$(11)$ & $\grave{n}$ & si & cóóríra & díi & i & bóló. \\
\hline & $1 S G$ & FH & exorcisme.ART & donner & 2SG & envers \\
\hline \multicolumn{7}{|l}{} \\
\multicolumn{7}{|l}{ 'Je t'apprendrai un exorcisme'. } \\
\hline
\end{tabular}

\begin{tabular}{|l|l|l|l|l|l|}
\hline (12) & kúnuín & mo & kà & náa & fagà \\
\hline & hier & $1 \mathrm{PL}$ & PST.TR & quatre.ART & mourir \\
\hline & \multicolumn{6}{|l}{ 'Hier nous avons tué quatre (personnes)'. } \\
\hline
\end{tabular}

MP bátí, bélé ...(-la) et kání qui ont deux syllabes chacun se prononcent avec le ton haut dans les deux contextes :

\begin{tabular}{|c|c|c|c|c|}
\hline (13) & búwmats & báti & 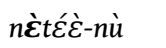 & dámú. \\
\hline & enceinte-ART & PRF & nété.ART-PL & manger \\
\hline
\end{tabular}


'La femme enceinte a mangé les grains de l'arbre néré'.

\begin{tabular}{|c|c|c|c|c|c|}
\hline \multirow{2}{*}{ (14) } & tui & ba $a^{-} t^{\prime}$ & huirgo & be ilebe -ile & $s a^{i-i ́ a ́}$ \\
\hline & souris-PL & PRF & palissade & grand & mettre \\
\hline & LS & & & palissade'. & \\
\hline
\end{tabular}

\begin{tabular}{|l|l|l|l|l|l|}
\hline$(15)$ & a'́illá & $k^{\prime}$ & á & fóo & do'Gódó \\
\hline & Dieux & PST.TR & $3 S G$ & dire & quelqu'un \\
\hline & \multicolumn{3}{|l}{ 'Dieu a dit que personne } \\
\hline
\end{tabular}

\begin{tabular}{|c|c|c|c|c|}
\hline kân & yع́ge & $b i$ tà & siómitî̀ & $t 0$ \\
\hline \multirow[t]{2}{*}{ IMP.NEG } & poisson & attraper & samedi.ART & dans \\
\hline & \multicolumn{4}{|c|}{ 'ne peut pêcher le samedi'. } \\
\hline
\end{tabular}

Ils s'est avéré que les MP maa et tee possèdent un ton haut aussi :

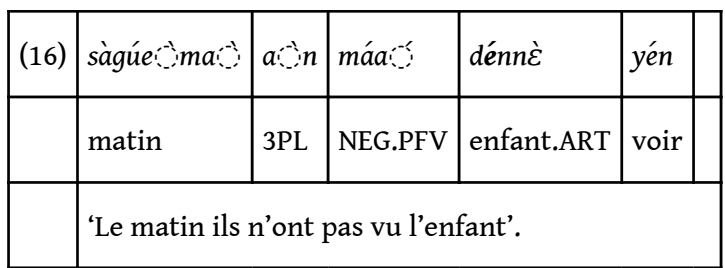

\begin{tabular}{|c|c|c|c|c|c|}
\hline (17) & $\grave{n}$ & maíáa & غ̇llà & yerin & buiturén \\
\hline & $1 \mathrm{SG}$ & NEG.PFV & mal & voir & encore \\
\hline & je & & & & leur \\
\hline
\end{tabular}

\begin{tabular}{|c|c|c|c|c|}
\hline (18) & $n$ & teise & 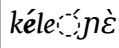 & dàmuì \\
\hline & $1 \mathrm{SG}$ & NEG.PFV & un.ART & manger \\
\hline
\end{tabular}

\begin{tabular}{|c|c|c|c|c|c|}
\hline$i$ & ko: & háaí: & $i$ & $n i i^{-5}$ & nàa. \\
\hline $2 S G$ & derrière & jusqu'à & $2 S G$ & OPT & venir \\
\hline
\end{tabular}


17 Cela signifie que l'unité quantitative qui définit le comportement tonal d'un MP n'est pas une syllabe mais une more. Ainsi, la règle de la réalisation du ton des MPs peut être formulée de la manière suivante :

(19) les MP à deux mores (minimum) ont un ton haut ;

les MP à une more ont un ton qui se polarise par rapport au ton de la syllabe suivante.

\subsection{Les formes réduites de MP}

Un autre argument en faveur de l'assertion que le comportement tonal des MP est déterminé par le nombre de leurs mores est la réalisation tonale des formes réduites de MP.

Ces formes existent pour tous les MP dissyllabiques : bélé, bátí et kání. Quand ils sont suivis par un mot commençant par une consonne, leur voyelle finale peut s'élider. Ces formes réduites sont facultatives, elles sont utilisées surtout dans le discours rapide. Dans le cas du MP kání il s'agit d'une simple omission de la voyelle finale, kání $\rightarrow$ kán :

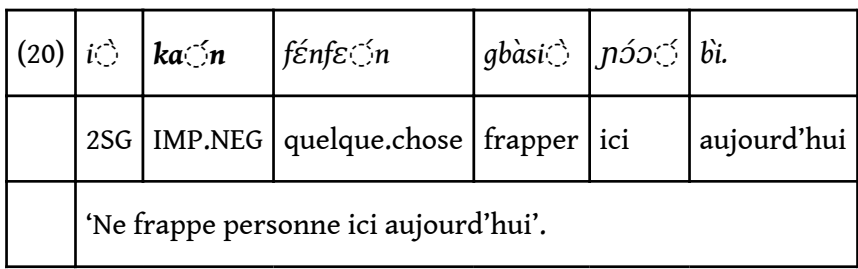

Quant aux MP bélé et bátí, leur réduction suit un modèle plus complexe. En général en kakabé, seule la consonne nasale peut apparaître dans la coda d'une syllabe (comme dans le cas de kání). Pour cette raison, la deuxième syllabe entière (plutôt que la voyelle finale) des MP bélé et bátí est élidée. En même temps, cette réduction n'est pas complète, car elle engendre la gémination de la consonne suivante :

bátí $+\mathrm{C} \rightarrow$ bá $\mathrm{C}:$

bélé $+\mathrm{C} \rightarrow$ bé $\mathrm{C}$ :

Dans les exemples (21) et (22) le nom dénc̀ appartenant au groupe nominal du complément d'objet direct et le verbe kinnógo se prononcent avec une consonne initiale géminée (la gémination est marquée par l'apostrophe dans la notation que j'utilise) :

\begin{tabular}{|c|c|c|c|c|}
\hline (21) & $a a^{-1}$ & ba & déjǹ & sòtso- \\
\hline & $3 S G$ & PRF & enfant.ART & recevoir \\
\hline & & & anfant'. & \\
\hline
\end{tabular}

\begin{tabular}{|l|l|l|l|l|l|l|}
\hline$(22)$ & i & sí & kálá, & á & kinnógó-ndén & dé, \\
\hline
\end{tabular}




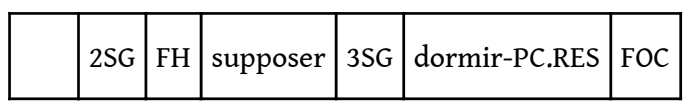

\begin{tabular}{|c|c|c|c|}
\hline háriós & $a a^{-1}$ & $b e^{i^{-\vdots}}:$ & kinnógó-nde \\
\hline mais & $3 S G$ & NEG.IPFV & dormir-PC.RES \\
\hline & \multicolumn{3}{|c|}{ 'Tu penses qu'elle dort, mais elle ne dort pas'. } \\
\hline
\end{tabular}

Bien qu'il existe un certain degré de fusion phonologique entre les MP bélé et báti et le morphème suivant, syntaxiquement ces MP possèdent une autonomie importante par rapport au constituant suivant. Si on prenait la gémination comme un argument pour imposer un statut préfixal aux MPs, on serait obligé de dire que ce sont des préfixes qui s'attachent à tout l'ensemble du groupe verbal. On reviendra sur la question d'autonomie morphologique d'un MP dans la section 2 où les formes contractées « MP + pronom » sont décrites en détail.

L'utilisation des formes réduites de bélé et bátí varie selon le dialecte. La forme réduite de bélé n'existe que dans les dialectes sud-est. Quant à baí', bien qu'attestée dans les deux groupes de dialectes, son utilisation n'est possible dans les dialectes nord-ouest que devant les mots à consonnes initiales dentales ( $t$ ou $d)$, eg. : bátí tágá 'PRF aller' $\rightarrow$ bá' tágá [báttágá].

Dans les dialectes sud-est bátí et bélé peuvent être réduits devant n'importe quelle consonne ${ }^{2}$, par exemple :

\begin{tabular}{|c|c|c|c|c|c|}
\hline (23) & $a n$ & $b a$ & yع́gॄ & bitat & háa \\
\hline & 3PL & PRF & poisson & attraper & jusqu’à \\
\hline
\end{tabular}

\begin{tabular}{|c|c|c|c|c|c|c|}
\hline a)n & na:- & sáa & ba & fáa & yéges: & là. \\
\hline $3 \mathrm{PL}$ & POSS & sac-ART & PRF & remplir & poisson.ART & LOC \\
\hline
\end{tabular}

Les formes réduites de bélé, báti et káni gardent leur ton haut quel que soit le ton du mot suivant. Comme ces formes sont représentées par des syllabes fermées, c'est-à-dire contenant deux mores chacune, leur ton haut est expliqué par la règle (19) : les MP à deux mores portent un ton haut, et les MPs à une more ont un ton polarisant.

Dans les dialectes nord-ouest, outre la réduction bátí $\rightarrow$ bá, une élision de la première syllabe (báti $\rightarrow t i$ ) peut se produire dans tout contexte phonologique :

\begin{tabular}{|c|c|c|c|c|}
\hline (24) & $6 a$ & 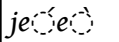 & bá' & tấgá, \\
\hline & quand & eau.ART & PRF & aller \\
\hline
\end{tabular}




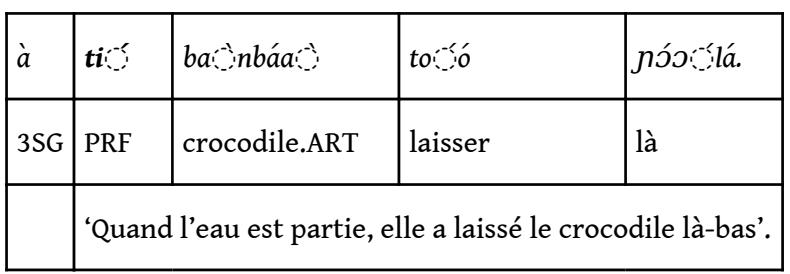

À la différence de bá', le ton de ti se polarise :

\begin{tabular}{|c|c|c|c|c|c|c|c|c|c|c|}
\hline (25) & álla & $b a a^{\prime} t^{\prime}$ & 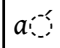 & dècima & $a-$ & $t i i^{-}$ & kínáng & sذ̀to & wóว & lún. \\
\hline & Dieu & PRF & $3 S G$ & aider & $3 S G$ & PRF & dîner.ART & obtenir & ce & jour \\
\hline
\end{tabular}

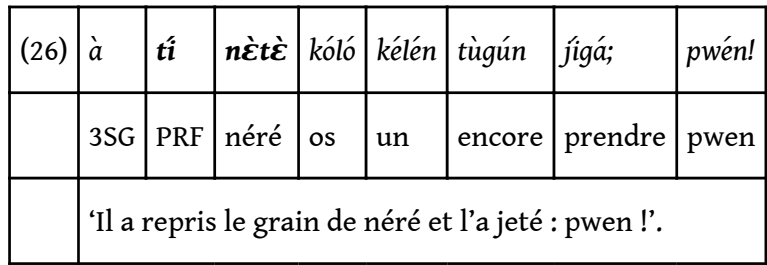

La différence de comportement tonal des allomorphes bá' et $t i$ s'explique par le fait que ti ne comporte qu'une seule more.

\subsection{Autres cas de polarisation du ton en kakabé}

Dans ce qui suit, je considère d'autres cas de la polarisation tonale en kakabé.

Marqueur de possession. Le ton du marqueur de possession se polarise par rapport au nom suivant :

\begin{tabular}{|l|l|l|l|}
\hline$(27 a)$ & mùsée & lá & nìngée \\
\hline & femme.ART & POSS & vache.ART \\
\hline & 'la vache de la femme' \\
\hline
\end{tabular}

\begin{tabular}{|l|l|l|l|}
\hline$(27 b)$ & mùsée & là & kérè \\
\hline & femme.ART & POSS & houe.ART \\
\hline & \multicolumn{2}{|l}{} \\
\hline
\end{tabular}

Préfixes verbaux. Il existe trois préfixes verbaux en kakabé : la- marqueur du causatif ; ma- marqueur de la pluralité verbale; ta- marqueur du réfactif (la répétition de l'action). Leur ton est toujours l'inverse du ton de la première syllabe de la base verbale : 
là-fátán CAUS-diviser 'diviser' - la -'-bo yi CAUS-tomber 'laisser tomber' ;

ta - ta

$m \boldsymbol{a}$-jélé VERB.PL-rire 'rire beaucoup' - ma grande quantité'.

Pronoms. Ci-dessous sont représentés les pronoms personnels kakabé dans les deux groupes de dialectes :
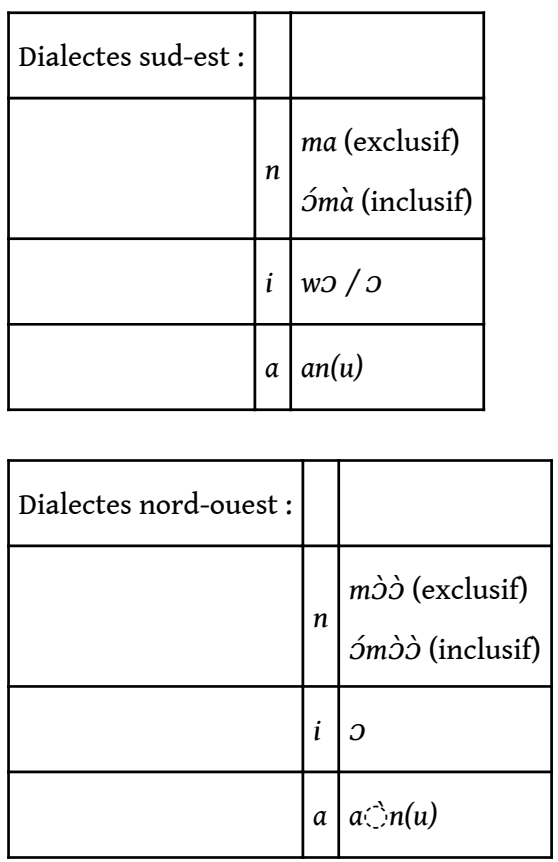

32 Il n'y a que trois pronoms de ton lexical stable : 1) le pronom inclusif de la première personne ómò̀̀ (dialectes nord-ouest) et ómà (dialectes sud-est) ; 2) le pronom pluriel de la troisième personne ànù ; 3) le pronom pluriel exclusif de la première personne mò̀̀ (dialectes nord-ouest).

Les tons de tous les autres pronoms se polarisent par rapport au ton lexical du mot suivant :

\begin{tabular}{|l|l|l|l|l|}
\hline ǹ/i/à/̀̀/ & mà tólè ‘ & mon & oreille' & ń/îá/ó/ má jùsée 'mon/ton/son/votre cœur' \\
\hline B & H & H & B & \\
\hline
\end{tabular}

\begin{tabular}{|c|c|c|c|}
\hline$n / /$ & $a / \partial / m a$ & táGátá & 'je suis allé $n$ \\
\hline B & $\mathrm{H}$ & $\mathbf{H}$ & B \\
\hline
\end{tabular}

On peut donc conclure que la tonalité des pronoms personnels dépend de la quantité de mores, et que tous les pronoms monomores portent un ton polarisé. Il y a une précision à faire ici. La polarisation du ton du pronom singulier de la troisième personne $a$ n'est pas obligatoire devant un ton bas. Ainsi, on a des variantes libres á kàran et à kàran 'lis !' 

protomokolé (ils sont différents dans les langues mandingues). En koranko et lélé (deux autres langues qui appartiennent à mokolé) les pronoms personnels ont les formes suivantes.

\begin{tabular}{|l|l|}
\hline \multicolumn{2}{|l|}{ koranko (Kastenholz 1987: 172) : } \\
\hline$n ́$ & mà \\
\hline$\grave{i}$ & wó \\
\hline$\grave{a}$ & ànu \\
\hline
\end{tabular}

\begin{tabular}{|c|c|}
\hline \multicolumn{2}{|c|}{ lélé (Vydrin 2009 : 39) : } \\
\hline$n$ & $n \hat{\prime}, m a ̀$ \\
\hline$\hat{i}$ & wó \\
\hline$\grave{a}$ & àno \\
\hline
\end{tabular}

On peut supposer que la tonalité des pronoms personnels du kakabé résulte d'un processus d'homogénisation. Les tons des pronoms monosyllabiques sont devenus polarisés avec l'exception que le pronom $a$ a gardé les traces du ton bas qu'il avait à l'origine.

\subsection{MP devant le verbe.}

Il a été montré que le ton d'un MP est soit haut soit polarisant en fonction de la quantité de mores que le MP contient. Mais les particularités du comportement tonal des MPs ne se limitent pas à cela. La règle (19) est valable pour les contextes où le MP est suivi immédiatement par un nom en fonction du complément d'objet direct. Quant aux constructions intransitives où le MP est suivi directement par le verbe, cette règle n'est respectée que partiellement. Le fait est que le ton lexical du verbe peut être facultativement remplacé par un ton bas, ce qui amène une neutralisation de son ton lexical.

Les exemples (28) - (33) montrent la variabilité des réalisations tonales des verbes tágá 'aller', bóo' ‘sortir' et fóló 'commencer' dont le ton lexical est haut ${ }^{3}$. On peut aussi remarquer que le nombre des mores du MP précédent n'a aucune importance : 


\begin{tabular}{|l|l|l|l|l|l|}
\hline$(28)$ & i & tî́ & fòlo & a & ba isàn-nà. \\
\hline & 2 SG & PRF & commencer & $3 S G$ & mélanger-GER \\
\hline & $\begin{array}{l}\text { 'Tu as commencé à les mélanger' (un des interlocuteurs accuse l'autre d'avoir commencé à } \\
\text { mélanger deux langues, le poular et le kakabé). }\end{array}$ \\
\hline
\end{tabular}

\begin{tabular}{|l|l|l|l|l|l|l|l|l|l|}
\hline$(29)$ & àn & $n i$ : & fólo & tún & à & dàmú-lá, & àn & ní & bità. \\
\hline & 3PL & OPT & commencer & juste & 3 SG & manger-GER & 3PL & OPT & attraper \\
\hline
\end{tabular}

\begin{tabular}{|c|c|c|c|c|c|c|c|}
\hline (30) & kúmuíkólè-nù & ‘ & úume-lén & tún, & àn & téé & bว̀̀े. \\
\hline & abeille.ART-PL & être & faire.du.bruit-PC.RES & juste & 3PL & NEG.FH & sortir \\
\hline & \multicolumn{7}{|c|}{ 'Ces abeilles-là font du bruit seulement, mais elles ne sortent pas'. } \\
\hline
\end{tabular}

\begin{tabular}{|c|c|c|c|c|c|c|c|c|}
\hline (31) & $\grave{n}$ & téé & bóó & nùn & 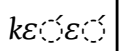 & kíla & là & few ! \\
\hline & $1 S G$ & NEG.FH & sortir & RETR & ce & chemin.ART & LOC & complètement \\
\hline & \multicolumn{8}{|c|}{ 'Je ne serais jamais sorti sur cette route (si j'avais su qu'on se rencontrerait)'. } \\
\hline
\end{tabular}

\begin{tabular}{|c|c|c|c|c|c|c|}
\hline (32) & dóe & $b a a^{\prime}$ & tágá & jii & sòri@ & dúla \\
\hline & celui & PRF & aller & eau & puiser & place \\
\hline & \multicolumn{6}{|c|}{ 'Il est allé puiser de l'eau'. } \\
\hline
\end{tabular}

\begin{tabular}{|c|c|c|c|c|c|c|c|c|}
\hline (33) & $6 a a^{-} y$ & $\grave{a}$ & $b a a^{\prime}$ & tầa & $\grave{a}$ & $k^{\prime}$ & á & dògòn. \\
\hline & quand & $3 S G$ & PRF & aller & $3 S G$ & PST.TR & $3 S G$ & se.cacher \\
\hline & \multicolumn{8}{|c|}{ 'Il est allé se cacher'. } \\
\hline
\end{tabular}

Conformément à la première partie de la règle (19), le ton d'un MP à deux mores reste toujours haut, cf. (31-33). Quant aux MP monomores, selon la deuxième partie de (19), ils doivent se polariser par rapport au verbe. C'est justement le cas de la réalisation tonale du MP devant un verbe à ton lexical bas. Il se trouve que dans ce cas le ton du MP est toujours haut, il est donc toujours en contraste avec le ton du verbe, par exemple :

\begin{tabular}{|l|l|l|l|l|}
\hline (34) & mò̀ & tî́ & bèn & yán. \\
\hline
\end{tabular}




\begin{tabular}{|l|l|l|l|l|}
\hline & $1 \mathrm{PL}$ & PRF & rencontrer & ici \\
\hline & \multicolumn{3}{|l}{ 'Nous nous sommes rencontrés ici'. } \\
\hline
\end{tabular}

La situation avec des verbes à ton lexical haut et plus compliquée. Comme ce dernier est variable, on peut imaginer trois scénarios différents de la réalisation du ton du MP : 1) soit le ton du MP se polarise par rapport au ton lexical du verbe, quelle que soit sa réalisation superficielle 2) soit le ton du MP se polarise par rapport au ton de surface du verbe, quel que soit son ton lexical 3) soit, vue l'instabilité de sa réalisation, le ton du verbe ne sert pas de repère pour le ton du MP.

Ce qui est attesté dans les données du corpus du kakabé, c'est une irrégularité de réalisation tonale des MP monomores. Par exemple, si le verbe à ton lexical haut est prononcé avec un ton bas, le ton de MP peut être soit haut (35) soit bas (36) :

\begin{tabular}{|l|l|l|l|l|l|}
\hline (35) & \multicolumn{4}{|l|}{ máá 'faire', ton lexical haut } \\
\hline & anu & kó, & sùmúnè & sí & màa. \\
\hline & 3PL & dire & soirée.ART & FH & faire \\
\hline
\end{tabular}

\begin{tabular}{|c|c|c|c|c|c|c|}
\hline \multirow[t]{2}{*}{ (36) } & \multicolumn{6}{|c|}{$k \varepsilon$} \\
\hline & dònع́غ̇ & ni - & $k \dot{\varepsilon} \varepsilon^{\prime}$ & jinbe & $n i$ & gbàsi \\
\hline & danser.ART & OPT & faire & tambour.jimbé.ART & OPT & frapper \\
\hline & \multicolumn{6}{|c|}{ '(Quand les parents viennent), on danse, on joue du tambour'. } \\
\hline
\end{tabular}

Si le verbe garde son ton haut, le MP à une more est prononcé le plus souvent avec un ton bas comme dans les exemples (29) et (30). Mais un ton haut est aussi possible dans ce contexte :

\begin{tabular}{|c|c|c|c|c|c|c|}
\hline (37) & $a ́ n$ & $n \hat{i}$ & $m \varepsilon \varepsilon^{-\zeta}$ & лว́ว & $n \varepsilon^{-1}$ & donde \\
\hline & $3 \mathrm{PL}$ & OPT & attendre & ici & dans & un.peu \\
\hline & \multicolumn{6}{|c|}{ 'Ils attendent là un peu'. } \\
\hline
\end{tabular}

\begin{tabular}{|l|l|l|l|l|l|l|}
\hline (38) & î̀ & si & kóo & wóte & to & tùguìn. \\
\hline & $2 \mathrm{SG}$ & $\mathrm{FH}$ & donner & argent.ART & dans & Encore \\
\hline \multicolumn{6}{|c|}{ 'Tu distribues de l'argent de nouveau'. } \\
\hline
\end{tabular}




\begin{tabular}{|l|l|l|l|l|l|}
\hline$(39)$ & $\grave{n}$ & $\boldsymbol{b i} i$ & wálí-lá & $\grave{j}$ & yén. \\
\hline & $1 \mathrm{SG}$ & être & travailler-GER & 2PL & pour \\
\hline \multicolumn{7}{|l}{ 'Je travaille pour vous'. } \\
\hline
\end{tabular}

Les chiffres des occurrences des MP avec un ton bas ou avec un ton bas dans la position devant un verbe dans mon corpus de textes sont représentés dans le Tableau 2 :

Tableau 2. Ton sur le MP dans la position devant des verbes à ton lexical haut et à ton lexical bas

\begin{tabular}{|l|l|l|l|}
\hline \multicolumn{3}{|l|}{ ton du verbe } \\
\hline ton du MP & ton lexical bas & ton lexical haut préservé & ton lexical haut abaissé \\
\hline ton bas & $0(0 \%)$ & $21(64 \%)$ & $13(62 \%)$ \\
\hline ton haut & $26(100 \%)$ & $12(36 \%)$ & $8(38 \%)$ \\
\hline total & $26(100 \%)$ & $33(100 \%)$ & $21(100 \%)$ \\
\hline
\end{tabular}

Malheureusement, le nombre des occurrences de MPs monomores dans le contexte devant verbe n'est pas très important dans mon corpus de textes. Ce n'est pas étonnant, puisqu'il s'agit de contextes qui doivent correspondre à plusieurs conditions en même temps : ce doit être une construction intransitive avec le MP bi, si, ni ou ti (une des formes réduites du MP bátí), le MP ka ne faisant pas partie de cette liste car il n'est utilisé que dans des constructions transitives. Au total, j'ai trouvé 80 occurrences de la construction dans laquelle un MP monomore est suivi d'un verbe, et dans 54 cas le verbe a un ton lexical haut. Cependant, les données du tableau permettent de tirer certaines conclusions par rapport à l'opposition entre les tons lexicaux haut et bas du verbe. Dans les 26 occurrences devant le verbe à ton lexical bas, le MP est prononcé avec un ton haut. Par contre, si le verbe a un ton lexical haut (soit préservé soit abaissé), le ton du MP est arbitraire, avec les quatre combinaisons possibles attestées dans les données :

\begin{tabular}{|l|l|l|}
\hline (1) ton bas (MP) & $\rightarrow$ ton bas (verbe) & 21 occurrences \\
\hline (2) ton haut (sur MP) & $\rightarrow$ ton bas (verbe) & 12 occurrences \\
\hline (3) ton bas (MP) & $\rightarrow$ ton haut (verbe) & 13 occurrences \\
\hline (4) ton haut (MP) & $\rightarrow$ ton haut (verbe) & 8 occurrences \\
\hline
\end{tabular}

47 Cela montre que l'opposition entre des verbes à ton lexical haut et à ton lexical bas n'est que partielle : si le verbe a un ton lexical bas, le ton de MP est toujours haut, bien qu'avec les verbes à ton lexical haut, trois autres configurations sont possibles dont seule la configuration (2) est ambiguë. Par exemple, dans l'énoncé sùmúnè sì màa 
(exemple (35)) le ton bas du MP si indique que le ton lexical du verbe est haut: un MP monomore ne peut pas porter un ton bas devant un verbe à ton lexical bas. Ainsi, le ton lexical du verbe n'est pas neutralisé complètement, mais cette opposition tonale se manifeste sur le MP précédent. Il n'est pas clair de comprendre pourquoi la polarisation du ton du MP n'est pas obligatoire devant le verbe à ton lexical haut. De plus, la question demeure de savoir quelles sont les conditions exactes de l'abaissement du ton lexical haut du verbe.

Ont donc été examinées les réalisations tonales sur les MPs et les verbes qui les suivent immédiatement. Les résultats sont synthétisés dans le Tableau 3 :

Tableau 3. Ton du MP et le ton du lexème qui le suit

\begin{tabular}{|l|l|l|l|}
\hline contexte & $\begin{array}{l}\text { quantité de mores } \\
\text { du MP }\end{array}$ & ton du MP & $\begin{array}{l}\text { ton du lexème qui } \\
\text { suit le MP }\end{array}$ \\
\hline \multirow{2}{*}{ MP + nom } & deux mores & haut & lexical \\
\cline { 2 - 5 } & une more & polarisant & lexical \\
\hline \multirow{2}{*}{$\begin{array}{l}\text { MP } \\
\text { verbe }\end{array}$} & deux mores & haut & lexical / bas \\
\cline { 2 - 5 } & une more & $\begin{array}{l}\text { polarisant devant le ton lexical B / H ou B } \\
\text { devant le ton lexical H }\end{array}$ & lexical / bas \\
\hline
\end{tabular}

Dans ces deux contextes le ton du MP est influencé par le ton du lexème qui le suit. Le MP à deux mores a toujours un ton haut. Le ton du nom est stable dans la position après $\mathrm{MP}$, et si ce dernier consiste en une seule more, son ton est obligatoirement polarisé par rapport au ton du nom. Le ton lexical du verbe après le MP peut être neutralisé en faveur du ton bas. Si le ton lexical du verbe est bas, le ton du MP à une more se polarise toujours par rapport à lui. Par contre, si le ton lexical du verbe est haut, le MP peut porter soit un ton haut soit un ton bas.

\section{Le MP et le pronom}

\subsection{Le pronom précédant le MP}

Comme cela a été mentionné dans la Section 1, le ton des pronoms à une seule more se polarise devant un verbe ou un nom. De la même manière, il est polarisé devant un MP à deux mores, et, comme tous les MP à deux mores ont un ton lexical haut, le pronom est réalisé avec un ton bas.

\begin{tabular}{|c|c|c|c|}
\hline (40) & $n$ & be & $m \varepsilon \varepsilon^{-1}-\operatorname{la}$ \\
\hline & $1 S G$ & NEG.IPFV & durer.longtemps-GER \\
\hline & \multicolumn{3}{|c|}{ 'Je ne resterai pas longtemps’. } \\
\hline
\end{tabular}




\begin{tabular}{|c|c|c|c|c|c|}
\hline (41) & $a$ & $b a a^{-} t^{\prime}$ & 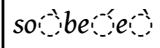 & naiàti & yánne \\
\hline & $3 S G$ & PRF & viande.ART & apporter & ici \\
\hline & & & la $v$ & à-bas'. & \\
\hline
\end{tabular}

51 Mais si le pronom monomore est devant un MP à une more, son ton ne se polarise pas. Il est toujours bas, quel que soit le ton du MP :

\begin{tabular}{|l|l|l|l|l|l|l|l|}
\hline$(42)$ & $\mathbf{i}$ & sì & kínè & tà-tábí & ì & sì & wótè \\
\hline & $2 S G$ & FH & repas.ART & REP-préparer & 2 SG & aFH & argent.ART \\
\hline & tà-Ókkítíré. \\
\hline & REP-distribuer \\
\hline & 'Tu prépareras le repas et tu distribueras l'argent'. \\
\hline
\end{tabular}

\begin{tabular}{|l|l|l|l|l|l|}
\hline$(43)$ & $\mathbf{i}$ & bi & nàá-lá & mùn & tùmà ? \\
\hline & 2 SG & être & venir-GER & quel & temps \\
\hline \multicolumn{4}{|l}{} \\
\multicolumn{4}{|l}{ 'Quand est-ce que tu viendras?’ } \\
\hline
\end{tabular}

52 Tout cela suggère l'existence d'une règle qui détermine le comportement tonal des séquences formées de plusieurs morphèmes monomores :

Dans une séquence de morphèmes grammaticaux monomores qui précède un morphème à (44) ton lexical, le dernier morphème a un ton polarisant par rapport à ce ton lexical, et tous les autres morphèmes reçoivent un ton bas.

Voici des exemples avec une séquence de trois morphèmes grammaticaux monomores, composé d'un pronom, d'un MP et d'un préfixe verbal :

\begin{tabular}{|l|l|l|l|}
\hline$(45)$ & $\grave{i}$ & sì & tà-tágámán. \\
\hline & 2 SG & FH & REP-se.promener \\
\hline & \multicolumn{3}{|c|}{ 'Tu iras te promener de nouveau'. } \\
\hline
\end{tabular}

\begin{tabular}{|l|l|l|l|}
\hline$(46)$ & i & sì & tá-nàà. \\
\hline & $2 S G$ & FH & REP-venir \\
\hline
\end{tabular}




\subsection{Le pronom suivant un MP : les formes contractées}

\subsection{1.}

Quand un MP est suivi d'un pronom personnel (en fonction de complément d'objet direct), une contraction peut se produire, se manifestant par une modification de la qualité de la voyelle et l'apparition d'un contour tonal spécial.

À l'exception du $n$ 1SG et mo ó́ / ma 1PL, les pronoms personnels du kakabé ont une voyelle à l'initiale, et tous les MP ont une voyelle en finale. En se combinant, la voyelle finale du MP et la voyelle initiale du pronom fusionnent en une voyelle longue dont la qualité est celle de la voyelle du pronom.

Dans le Tableau 4, les formes contractées avec les MP bi, tée, máa et béle sont présentées :

Tableau 4. Formes contractées des MP bi, tée, máa, béle et des pronoms personnels

\begin{tabular}{|c|c|c|c|c|c|c|}
\hline & $2 \mathrm{SG}$ & $3 S G$ & 1PL.INCL & $2 \mathrm{PL}$ & $3 \mathrm{PL}$ \\
\hline & & $i$ & $A$ & วтวо & 0 & an $(u)$ \\
\hline être & $b i$ & $b^{\prime} i i$ & $b^{\prime} a a$ & b' ววтวว & b' วอ & b'aanu \\
\hline NEG.FH & téé & $t^{\prime} i i$ & $t^{\prime} a a$ & t' วอтวว & $t^{\prime}$ วอ & t'aanu \\
\hline NEG.PFV & máá & $m^{\prime} i i$ & $m^{\prime} a a$ & m' ววтวว & m' วอ & m'aanu \\
\hline NEG.être & bélé & bel' ii / bil' ii & bal' aa & bel' วอтวว & bel' ว & bal' aanu \\
\hline
\end{tabular}

La longueur de la voyelle ne compte pas : tée et máá ont des formes contractées de la même manière que bi et bélé.

En combinaison avec les pronoms A, àn(ù) et $i$, le MP bélé change ses deux voyelles (dans le cas du pronom $i$, l'assimilation de la première voyelle de bélé est facultative).

Les formes contractées de tous les MP avec les pronoms $i$, $a$ et anu sont presque obligatoires. La forme non-contractée n'apparait qu'en prononciation ralentie ou s'il y a une pause d'hésitation après le MP. Par contre, les formes contractées avec les pronoms $\supset$ et $\supset$ moว sont plutôt facultatives.

La question se pose du statut morphologique des formes contractées. On pourrait supposer que l'existence d'une contraction phonologique en kakabé entre un MP et un pronom indique que ces deux unités ne sont pas des morphèmes libres mais plutôt des affixes.

Cette hypothèse n'est pas confirmée par la syntaxe. Le pronom qui suit immédiatement le verbe peut être soit un complément d'objet direct, soit un possesseur pronominal à l'intérieur du constituant d'objet direct. Et quel que soit son statut, la contraction se fait de la même manière : 


\begin{tabular}{|c|c|c|c|c|}
\hline (47) & Músá & $k^{\prime}$ & $\grave{a}$ & kذ̀ntว̀n \\
\hline & Moussa & PRF & $3 S G$ & saluer \\
\hline & \multicolumn{4}{|c|}{ 'Moussa l'a salué'. } \\
\hline
\end{tabular}

\begin{tabular}{|l|l|l|l|l|l|l|}
\hline (48) & Músá & $k$ ' & à & là & dénè & $k$ J̀ntòn \\
\hline & Moussa & PRF & POSS & 3SG & enfant.ART & saluer \\
\hline \multicolumn{6}{|l}{} \\
\hline \multicolumn{7}{|l}{ 'Moussa a salué son enfant'. } \\
\hline
\end{tabular}

61 Si l'on traitait les MP comme des morphèmes dépendants, on serait obligé de postuler pour le kakabé l'existence de préfixes qui s'attachent au constituant nominal d'objet direct avec tous ses dépendants ou, étant donné que les MPs ont un rapport sémantique plus proche avec le verbe qu'avec le complément d'objet direct, à tout l'ensemble du groupe verbal. Donc, malgré la contraction phonologique, je préfère considérer les MPs en kakabé comme des mots auxiliaires plutôt que des préfixes.

\subsubsection{Les formes contractées avec le MP bi}

Le MP bi manifeste un comportement spécial en ce qui concerne sa réduction. Premièrement, ce marqueur a une forme facultative sans consonne : $i$. Deuxièmement, il peut être omis facultativement. Cependant, l'omission de bi ou son allomorphe sans consonne ne sont pas possibles devant des pronoms qui ont des formes contractées:

\begin{tabular}{|l|l|l|l|l|l|l|}
\hline (49a) & Músaí: & $\boldsymbol{b}^{\prime}$ & áá & là & dénè & yén-ná. \\
\hline & Moussa & être & 3SG & POSS & enfant.ART & voir-GER \\
\hline \multicolumn{6}{|l}{} \\
\hline
\end{tabular}

\begin{tabular}{|l|l|l|l|l|l|l|}
\hline$(49 \mathrm{~b})$ & * Músá & $\grave{i}$ & $\grave{a}$ & là & dénè & yén-ná. \\
\hline & Moussa & être & 3SG & POSS & enfant.ART & voir-GER \\
\hline$(49 \mathrm{c})$ & * Músá & $\varnothing$ & $\grave{a}$ & là & dénè & yén-ná. \\
\hline & Moussa & être & 3SG & POSS & enfant.ART & voir-GER \\
\hline
\end{tabular}

Ainsi la forme contractée du MP apparaît chaque fois devant un pronom à voyelle initiale.

En fonction de copule existentielle, devant un pronom, bi ne peut pas être réduit à une voyelle :

\begin{tabular}{|l|l|l|l|l|l|l|}
\hline (50a) & jáámíyè & b' & áá & là & hódè & tò. \\
\hline
\end{tabular}




\begin{tabular}{|l|l|l|l|l|l|l|}
\hline & mosquée & être & $3 S G$ & POSS & village.ART & dans \\
\hline & 'Il y a une mosquée dans son village'. \\
\hline
\end{tabular}

(50b) * jáámíyè ì à là hódè tò.

Mais à la différence de bi en fonction de MP, la copule bi peut être omise devant la voyelle d'un pronom :

(51) ja Ǵámíyé o à là hódè tò. 'Il y a une mosquée dans son village’.

Les règles de réalisation du MP bi et de la copule bi sont comparées dans le Tableau 5.

Tableau 5. Variantes de réalisation de bi devant voyelle dans un énoncé verbal et non-verbal

\begin{tabular}{|l|l|l|}
\hline & $b i-$ copule existentielle & $b i-\mathrm{MP}$ \\
\hline forme réduite $i$ & non & non \\
\hline omission de $b i$ & + & non \\
\hline forme contractée & + & + \\
\hline
\end{tabular}

L'élision de la consonne de bi (soit comme copule existentielle, soit comme MP) devant un pronom à voyelle initiale est impossible pour des raisons phonologiques: dans le système phonologique du kakabé, il est exclu d'avoir trois voyelles de suite. Par contre, l'acceptabilité de l'omission de bi est réglementé par un facteur morphophonologique : elle est permise pour une bi-copule et interdite pour un bi-MP.

Apparemment, le lien syntaxique entre un MP et le pronom suivant est plus étroit que celui entre une copule et un pronom.

\subsection{Le contour tonal de la forme amalgamée «MP + pronom »}

La forme amalgamée d'un MP et d'un pronom reçoit un ton haut. Devant un autre ton haut, elle peut se prononcer avec un contour tonal descendant.

Ce contour tonal s'étend sur toute la séquence qui peut contenir une, deux ou trois syllabes :

b' î | b' îi

bél' îi ou bél' îi

bél' óómóó ou bél' óómòò

Quant au pronom sujet qui précède cette séquence, son ton est bas :

\begin{tabular}{|c|c|c|c|c|c|c|c|c|}
\hline (52) & $n$ & $k^{\prime}$ & $a$ & mén, & so - ber-ien & 'i & $i$ & bo: \\
\hline
\end{tabular}




\begin{tabular}{|l|l|l|l|l|l|l|l|l|}
\hline & 1SG & PST.TR & 3SG & entendre & viande.ART & être & 2SG & vers \\
\hline
\end{tabular}

Le contour tonal haut / ascendant est attribué à n'importe quelle séquence «MP + pronom objet" (sauf dans le cas où le locuteur fait une pause entre les deux

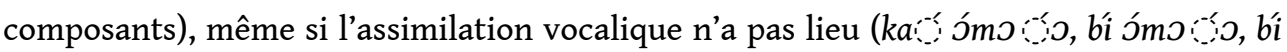
ó etc.). Dans ce contexte, la distinction tonale entre les MP à une more et ceux à deux mores se neutralise.

Quant au verbe, son ton lexical haut peut être abaissé de la même manière qu'après un MP (sans pronom objectif), cf. les exemples (53) et (54), les verbes dámú et kélé étant à ton lexical haut:

\begin{tabular}{|c|c|c|c|c|c|}
\hline (53) & $\grave{n}$ & $s^{\prime}$ & $i=i$ & dà̀ & kذ̀tદ́ċ ! \\
\hline & $1 S G$ & $\mathrm{FH}$ & $2 S G$ & manger & maintenant \\
\hline & \multicolumn{5}{|c|}{ 'Je te mangerai maintenant!' } \\
\hline
\end{tabular}

\begin{tabular}{|c|c|c|c|c|c|c|c|c|}
\hline (54) & mansais & $k^{\prime}$ & $a a_{0}^{\prime-1}$ & $k e$ ile & $\grave{a}$ & là & $t a^{0} \cdot n d \bar{\varepsilon}$ & tò. \\
\hline & chef.ART & PST.TR & $3 S G$ & appeler & $3 S G$ & POSS & cour.ART & dans \\
\hline & \multicolumn{8}{|c|}{ 'Le chef l'a appelé dans sa cour'. } \\
\hline
\end{tabular}

Et le ton lexical du nom reste stable.

72 Les données du corpus montrent que le ton lexical du verbe est abaissé presque deux fois plus souvent après une séquence "MP + pronom» (73\% d'occurrences), qu'immédiatement après un MP ( $40 \%$ d'occurrences) :

Tableau 6. Fréquence de la neutralisation du ton lexical haut

\begin{tabular}{|l|l|l|l|}
\hline & $\begin{array}{l}\text { le ton lexical haut du } \\
\text { verbe est préservé }\end{array}$ & $\begin{array}{l}\text { le ton lexical haut } \\
\text { du verbe est abaissé }\end{array}$ & $\begin{array}{l}\text { le nombre total d'occurrences } \\
\text { des verbes avec ton lexical } \\
\text { haut }\end{array}$ \\
\hline $\begin{array}{l}\text { après } \\
\text { combinaison MP } \\
\text { pronom }\end{array}$ & $25(27 \%)$ & $67(73 \%)$ & $92(100 \%)$ \\
\hline après un MP & $64(60 \%)$ & $42(40 \%)$ & $106(100 \%)$ \\
\hline
\end{tabular}

La position après une séquence «MP + pronom » est défavorable pour la préservation du ton haut.

Par contre, le ton lexical du verbe est toujours préservé après un nom : 


\begin{tabular}{|c|c|c|c|c|}
\hline (55) & súluíikè & bóo-ta & dùnbe & búuitos \\
\hline & hyène.ART & sortir-PST.INTR & ruche.ART & dans \\
\hline & \multicolumn{4}{|c|}{ 'L'hyène est sortie de la ruche' } \\
\hline
\end{tabular}

\begin{tabular}{|l|l|l|l|l|l|l|}
\hline (56) & sííkúlí-ndè & $\mathrm{t}^{\prime}$ & á & là & sásá-ndè & jígá. \\
\hline & bouc-DIM.ART & PRF & 3SG & POSS & sac-DIM.ART & prendre \\
\hline \multicolumn{4}{|l}{} \\
\multicolumn{4}{|l}{ 'Le petit bouc a prit son sac'. } \\
\hline
\end{tabular}

\section{Le comportement tonal des MP : conclusions}

La réalisation tonale du MP est déterminé (1) par le nombre de mores qu'il comporte et (2) par la catégorie morphologique du mot suivant: un pronom, un verbe ou un nom. En même temps, le ton du mot suivant le MP est susceptible de modifications. Les règles sont représentées dans le Tableau 7.

Tableau 7. Le ton du MP ou de la séquence «MP + pronom » et le ton du mot suivant

\begin{tabular}{|l|l|l|l|}
\hline \multirow{2}{*}{ contexte } & $\begin{array}{l}\text { nombre des mores du } \\
\text { MP / MP-pronom }\end{array}$ & ton du MP / MP-pronom & $\begin{array}{l}\text { ton du lexème suivant } \\
\text { le MP / MP-pronom }\end{array}$ \\
\hline & deux mores & haut & lexical \\
\cline { 2 - 5 } & une more & polarisant & lexical \\
\hline \multirow{2}{*}{\begin{tabular}{l} 
MP + verbe \\
\cline { 2 - 5 }
\end{tabular}} & deux mores & haut & lexical / bas \\
\hline $\begin{array}{l}\text { MP-pronom } \\
+ \text { nom }\end{array}$ & deux mores ou plus & holarisant devant ton lexical B & lexical / bas \\
\hline $\begin{array}{l}\text { MP-pronom } \\
+ \text { verbe }\end{array}$ & deux mores ou plus & haut / descendant & lexical \\
\hline
\end{tabular}

Résumons : le ton d'un MP monomore se polarise devant le nom ou le verbe suivant. Un MP à deux mores porte toujours un ton haut. La séquence « MP + pronom » (le MP soit à une more, soit à deux mores) se réalise toujours avec un ton haut ou un ton descendant. Le ton lexical du nom reste toujours inchangé. Par contre, pour le ton lexical du verbe, la position après un MP ou après la séquence « MP + pronom » est faible : son ton lexical haut peut être abaissé. Cet abaissement se produit plus souvent après la séquence «MP + pronom » qu'immédiatement après un MP. On peut supposer que la stabilité du ton du nom est due au fait que le nom comporte presque toujours un article référentiel. Cet 
article a la forme d'une voyelle antérieure $e$ avec un ton bas qui se suffixe au nom. Les particularités de sa réalisation sont décrites en (Vydrina 2007). Cet article, suffixé à la racine $\mathrm{du}$ nom, possède son propre ton bas qui semble servir d'écran bloquant l'application des règles tonales contextuelles.

Une autre conclusion est qu'en kakabé il y a une corrélation entre la structure segmentale du MP et la stabilité de son ton. Cette question sera considérée en détails cidessous.

\section{La corrélation entre la longueur, le ton et le degré de grammaticalisation en kakabé}

La longueur et le ton sont en kakabé des phénomènes liés. Parmi les manifestations de cette corrélation, on peut citer l'allongement de la voyelle finale du nom suite à l'adjonction de l'article. L'allongement se produit quand l'article est ajouté à un nom à contour tonal ascendant, si le relèvement tonal commence sur la dernière syllabe du nom : donsó - dònséế. 'chasseur', kùrùndá - kùrùndáa 'anneau'; tồnòko t0 inj̀k

Un autre cas est la longueur vocalique des noms et verbes monosyllabiques. En général la longueur vocalique des morphèmes monosyllabiques est en fonction de la classe grammaticale à laquelle ils appartiennent. On distingue trois types de morphèmes :

1. morphèmes lexicaux : racines nominales, adjectivales, verbales, adverbiales ;

2. lexèmes auxiliaires : pronoms, MP ;

3. morphèmes grammaticaux suffixés : marque du causatif, marque du pluriel.

Si un morphème monosyllabique est lexical, sa voyelle est longue : báá 'grand', kàá 'serpent', káá 'ruisseau', sòó 'être debout', dáá 'créer'.

La longueur des voyelles des morphèmes monosyllabiques auxiliaires (des MP et des pronoms) est déterminée lexicalement. Certains MP monosyllabiques et pronoms ont une voyelle brève ( $i$ SSG, a 3SG, ni OPT, ka PST.TR, si FH etc.), d'autres ont une voyelle longue (máá NEG.PFV, tée NEG.FH, mòj̀ 1PL).

81 Enfin, les affixes (tous monosyllabiques) ont une voyelle brève : la- marque causative, ma- marque de la pluralité verbale, ta- marque du réfactif, -ma suffixe du passif, etc.

La division des morphèmes entre ces trois groupes se manifeste aussi dans leur comportement tonal. Les morphèmes lexicaux ont leur propre ton lexical (même s'il se neutralise dans certains contextes). La tonalité des morphèmes auxiliaires est en fonction de leur structure segmentale. Ceux comportant deux mores ou plus ont un ton stable ; par contre, les morphèmes auxiliaires à une more sont caractérisés par un ton polarisant. Enfin, le ton des affixes est toujours déterminé par le contexte. Le ton des préfixes verbaux se polarise par rapport au verbe, et le ton des suffixes est une copie du ton de la syllabe précédente.

83 Il y a deux morphèmes auxiliaires monomores dont le ton n'est pas polarisant mais plutôt bas stable : c'est le marqueur du séquentiel kà dont on a parlé dans la section 1.2 et l'article référentiel -è, qui a été mentionné à la fin de la section 3. En plus, il y a le pronom personnel de troisième personne qui a deux variantes de réalisation dans n'importe quel contexte : avec un ton polarisant ou avec un ton bas. 

jacent sur les morphèmes monomores et de dire que l'opposition ton haut / ton bas est préservée dans ce cas. En même temps, il faut noter qu'il existe un fort déséquilibre en faveur du ton polarisant: il n'y a que deux morphèmes monomores à ton bas stable. Évidemment, en diachronie, ce n'étaient que des morphèmes à ton haut mais aussi des morphèmes à ton bas qui ont acquis un ton polarisant. On peut citer le cas du pronom personnel 2PL.EXCL ma qui a un ton polarisant en kakabe, mais un ton bas en lélé et koranko. (cf. la section 1.3).

En tout cas, on ne peut nier l'existence de corrélation entre le ton et la structure segmentale du morphème, et ces deux paramètres formels sont liés au degré de grammaticalité qui est un paramètre sémantique.

Il est significatif que la longueur des verbes monosyllabique se distingue de la longueur des noms monosyllabiques. Dans la section 3 il a été montré que le ton lexical du nom est plus stable que le ton lexical du verbe. Il s'avère que la longueur d'un verbe monosyllabique est aussi systématiquement distincte de la longueur d'un nom. La voyelle d'un nom monosyllabique est toujours considérablement plus longue qu'une voyelle brève. Par contre, un verbe monosyllabique, en principe, peut se prononcer avec une voyelle brève. Quant à la prononciation longue de sa seule voyelle, elle est plutôt "semi-longue ", c'est-à-dire que la voyelle d'un verbe monosyllabique est beaucoup moins longue que la voyelle d'un nom monosyllabique. La question de la réalisation phonétique de la longueur des monosyllabes est analysée en détails dans (Vydrina 2008).

87 Tout cela nous conduit à la conclusion que la perte progressive du ton lexical accompagne le processus de la grammaticalisation en kakabé. On peut comparer le fonctionnement du ton en kakabé avec le fonctionnement de l'accent dans les langues à accent. L'accent est caractéristique d'un mot autonome, et le passage d'une unité lexicale à la catégorie des clitiques et conséquemment des affixes est accompagné par sa perte. En kakabé, chaque unité autonome se caractérise par son propre contour tonal (un ton lexical), et le passage à la classe des morphèmes auxiliaires s'accompagne de la perte de ton lexical.

et al. 1991: 15) propose la définition suivante de la grammaticalisation: "With the term "grammaticalizaion" we refer essentially to an evolution whereby linguistic units lose in semantic complexity, pragmatic significance, syntactic freedom, and phonetic substance, respectively".

En accord avec cette définition, en kakabé la grammaticalisation sémantique d'une unité et le changement des ses propriétés distributives sont accompagnés par la perte du ton et de la longueur. 


\section{BIBLIOGRAPHIE}

DAVYDOV A., 2010, "Historical morphology of personal pronouns in Manding”, Personal pronouns in Niger-Congo languages. International workshop. Abstracts and papers. Working materials, St. Petersburg, September 13-15 2010, pp. 23-37.

HEINE B., CLAUDI U., HÜNNEMEYER F., 1991, Grammaticalization: A Conceptual Framework, Chicago, University of Chicago Press.

KASTENHOLZ R., 1987, Das Koranko. Ein Beitrag zur Erforschung der Nord-Mande-Sprachen, Dissertation, Köln, Mundus.

VYDRINE Valentin, 2009, « Esquisse de la langue lélé (groupe mokolé) », Mandenkan 45, pp. 29-104.

VYDRINA Alexandra, 2008, "Vowel length in the Kakabe language", Mandenkan 44, pp. 79-88.

VYDRINA Alexandra, 2007, «Réalisation d'article référentiel en kakabé », IV colloque sur la typologie et la grammaire pour jeunes chercheurs. Institut de recherche linguistique, St. Pétersbourg, 1-3 novembre 2007, Recueil d'articles. Nestor-Istoriya, St. Pétersbourg, pp. 56-59 [en russe].

\section{NOTES}

1. Les recherches ont été effectuées sur un corpus de textes spontanés que j'ai enregistrés au cours des missions de terrain en Guinée en 2008, 2009 et 2010. La durée totale des textes enregistrés est $153 \mathrm{mn}$. En dehors de textes spontanés, des données élicitées ont aussi été utilisées dans certains cas.

2. Dans ce contexte, n'importe quelle consonne kakabé peut être géminée. Dans les autres contextes, les consonnes $f, s, h, w, y$ ne peuvent pas être géminées.

3. Comme le ton lexical du verbe peut être neutralisé, pour le déterminer il a fallu enregistrer chaque verbe dans un contexte spécial. Pour les verbes intransitifs c'est la forme à suffixe -ta (le marqueur du prétérit) et pour les verbes transitifs, la forme de l'impératif.

\section{RÉSUMÉS}

L'article porte sur la corrélation entre 1) l'autonomie tonale et le poids phonologique d'un morphème, et 2) la grammaticalisation de son sens en kakabé. Dans cette langue les morphèmes à valeur grammaticale, comme des mors auxiliaires, des affixes verbaux et nominaux, etc., tendent à comporter une syllabe légère et ne pas avoir de ton lexical spécifique. Le plus révélateur pour la corrélation entre la phonologie et la fonction grammaticale d'un morphème est le cas des marques prédicatives poste-sujettes. Du point de vue syntaxique, ces marques occupent la position intermédiaire entre les affixes et des morphèmes plus autonomes. Au niveau phonologique, cela se reflète par le fait que le paradigme des marques prédicatives inclut à la fois des marques tonalement dépendantes ne comportant qu'une seule syllabe légère, et des marques qui ont un ton lexical et comportent plus qu'une syllabe. 
The paper deals with the question of the correlation between 1) tonal independence and phonological weight of a morpheme and 2) the grammaticality of its meaning in the Kakabe language. In Kakabe morphemes with grammatical meaning, such as auxiliaries, different verbal and noun affixes etc. tend to contain only one light syllable and to possess no lexical tone of their own. The case of the post-subject auxiliary markers, which is in the focus of the paper, is especially revealing of the correlation between phonology and grammatical function of a morpheme. Syntactically, these markers occupy an intermediate position between the defendant affixes and more free lexical morphemes. This is mirrored at the phonological level in the fact that the paradigm of auxiliaries comprises both tonally dependent markers consisting of only one light syllable and markers which have their own tone and consist of more than one syllable.

В СТАТЬЕ РАССМАТРИВАЕТС КОРРЕЛЯЦИЯ МЕЖДУ 1 ) ТОНАЛЬНОЙ АВТОНОМИЕЙ И ФОНОЛОГИЧЕСКИМ ВЕСОМ МОРФЕМЫ, А ТАКЖЕ 2) ЕЁ ГРАММАТИЧНОСТЬЮ В ЯЗЫКЕ КАКАБЕ. МОРФЕМЫ С ГРАММАТИЧЕСКИМ ЗНАЧЕНИЕМ - СЛУЖЕБНЫЕ СЛОВА, ГЛАГОЛЬНЫЕ И ИМЕННЫЕ АФФИКСЫ, И Т.Д. - ЧАЩЕ ВСЕГО СОСТОЯТ ИЗ ОДНОГО ЛЁГКОГО СЛОГА И НЕ ИМЕЮТ ЛЕКСИЧЕСКОГО ТОНА. ОСОБЕННО ПОКАЗАТЕЛЕН ДЛЯ КОРРЕЛЯЦИИ МЕЖДУ ФОНОЛОГИЕЙ И ГРАММАТИЧЕСКОЙ ФУНКЦИЕЙ МОРФЕМЫ СЛУЧАЙ ПОСТ-СУБЪЕКТНЫХ ВСПОМОГАТЕЛЬНЫХ СЛОВ. В СИНТАКСИЧЕСКОМ ОТНОШЕНИИ ЭТИ ПОКАЗАТЕЛИ ЗАНИМАЮТ ПРОМЕЖУТОЧНУЮ ПОЗИЦИЮ МЕЖДУ СВЯЗАННЫМИ АФФИКСАМИ И АВТОНОМНЫМИ ЛЕКСИЧЕСКИМИ МОРФЕМАМИ. НА ФОНОЛОГИЧЕСКОМ УРОВНЕ ЭТО ОТРАЖАЕТСЯ В ТОМ, ЧТО ПАРАДИГМА ПРЕДИКАТИВНЫХ ПОКАЗАТЕЛЕЙ ВКЛЮЧАЕТ И ТОНАЛЬНО ЗАВИСИМЫЕ ПОКАЗАТЕЛИ, СОСТОЯЩИЕ ИЗ ОДНОГО ЛЁГКОГО СЛОГА, И ДВУХ- И БОЛЕЕ-СЛОЖНЫЕ ПОКАЗАТЕЛИ, НЕСУЩИЕ СВОИ СОБСТВЕННЫЕ ТОНЫ.

\section{INDEX}

Keywords : Kakabe Language, Mokole Languages, Grammatical Tone, Lexical Tone, Light Syllable, Morphonology, Phonology, Grammaticalization

Thèmes : kakabé, langues mokolé

motsclesru ЯЗЫК КАКАБЕ, ЯЗЫКИ МОКОЛЕ, ГРАММАТИЧЕСКИЙ ТОН, ЛЕКСИЧЕСКИЙ ТОН, ЛЁГКИЙ СЛОГ, МОРФОНОЛОГИЯ, ФОНОЛОГИЯ, ГРАММАТИКАЛИЗАЦИЯ

Mots-clés : ton grammatical, ton lexical, syllabe légère, morphophonologie, phonologie, grammaticalisation

\section{AUTEUR}

\section{ALEXANDRA VYDRINA}

LLACAN, UMR 8135

INALCO / USPC

alexandra.vydrina@gmail.com 